\title{
Prediction of Solution Behavior via Calorimetric Measurements Allows for Detailed Elucidation of Polyoxometalate Transformation
}

Authors: Hrafn Traustason, ${ }^{\dagger}$ Haylie L. Lobeck, ${ }^{\ddagger}$ Mengyu Xu, ${ }^{\ddagger}$ Patrick A. Julien, ${ }^{\ddagger}$ Mateusz Dembowski, ${ }^{\dagger}$ Peter C. Burns*+‡

${ }^{\dagger}$ Department of Chemistry and Biochemistry, University of Notre Dame, Notre Dame, Indiana 46556, United States

${ }^{\ddagger}$ Department of Civil \& Environmental Engineering \& Earth Sciences, University of Notre Dame, Notre Dame, Indiana 46556, United States

\section{Supporting Information}

\section{Table of Contents}

S1. Synthesis

S2. Inductively Coupled Plasma - Optical Emission Spectrometry

S3. Thermogravimetric Analysis

S4. Electrospray Ionization - Mass Spectrometry

S5. Density Measurements

S6. Thermographs

S7. Thermochemical cycles

S8. In situ Raman Spectroscopy

S9. Small Angle X-ray Scattering

S10. Pictures of Crystals

S11. Nuclear Magnetic Resonance

S12. Separations

S13. Crystallographic Information

\section{S1. Synthesis}


The synthesis for LiNa- $U_{24} \mathrm{Pp}_{12}$ was as previously published. ${ }^{1} 10 \mathrm{~mL}$ of $0.5 \mathrm{M} \mathrm{UO}_{2}\left(\mathrm{NO}_{3}\right)_{2}, 10 \mathrm{~mL}$ of $30 \% \mathrm{H}_{2} \mathrm{O}_{2}$, and $12.5 \mathrm{~mL}$ of $0.2 \mathrm{M} \mathrm{Na}_{4} \mathrm{P}_{2} \mathrm{O}_{7}$ were added to a beaker, resulting in an immediate precipitation of light yellow solid. Into the beaker was titrated $2.38 \mathrm{M} \mathrm{LiOH}$ until pH $=9.3$ resulting in dissolution of the precipitate, vigorous bubbling, and a dark orange solution. The $\mathrm{pH}$ was adjusted daily to 7.0 for the next 7 days or until no change in $\mathrm{pH}$ was noticed. The beaker was left open and orange crystals formed in about 4 weeks.

\section{S2. Inductively Coupled Plasma - Optical Emission Spectrometry}

Table S1. Ratio of elements obtained from ICP-OES results.

\begin{tabular}{cccc}
\hline Compound & Li:U ratio & Na:U ratio & Li:Na ratio \\
\hline LiNa-U $_{24} \mathrm{Pp} 12$ & $0.99(4)$ & $1.02(4)$ & 0.98 \\
\hline
\end{tabular}

\section{S3. Thermogravimetric Analysis}

Thermogravimetric analyses were conducted for aliquots of each POM using a Mettler-Toledo TGA-DSC 1. For each measurement, crystals were placed in an Al crucible and heated to $750^{\circ} \mathrm{C}$ at a rate of $5^{\circ} \mathrm{C} / \mathrm{min}$ under $\mathrm{Ar}$ gas flowing at $50 \mathrm{~mL} / \mathrm{min}$. For analysis, mass loss by $450^{\circ} \mathrm{C}$ was used for LiNa- $\mathrm{U}_{24} \mathrm{Pp}_{12}$

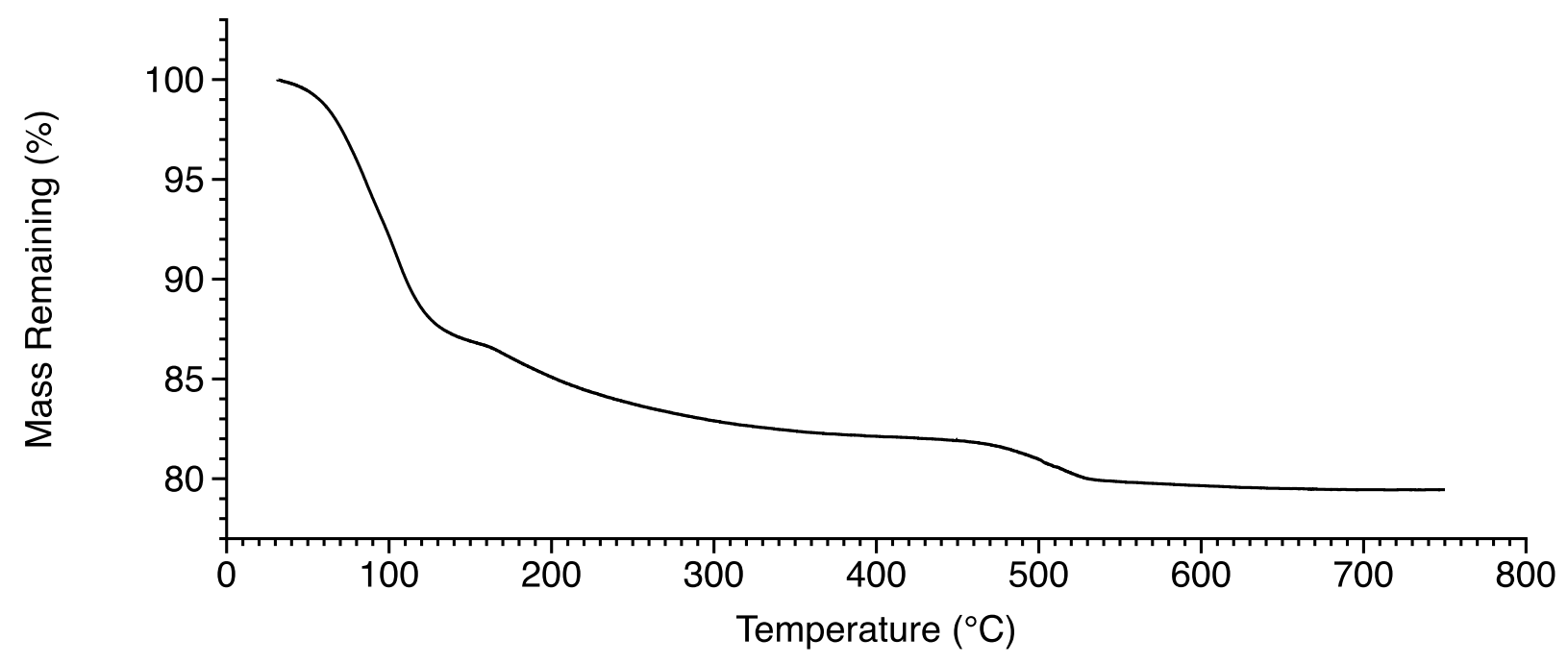

Figure S1. Thermogravimetric curve of $\mathrm{LiNa}-\mathrm{U}_{24} \mathrm{Pp}_{12}$. 


\section{S4. Electrospray lonization - Mass Spectrometry}

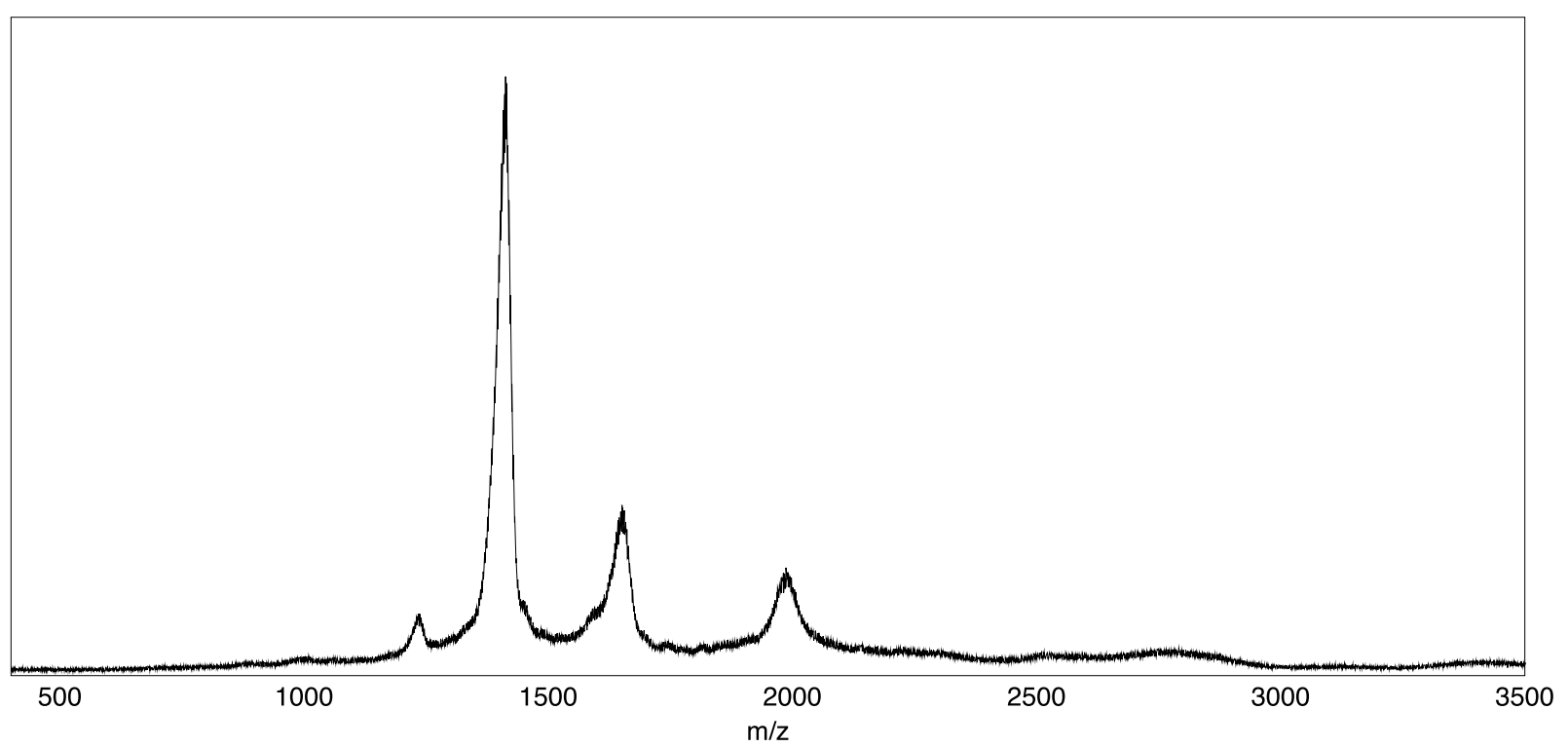

Figure S2. ESI-MS spectra of LiNa- $\mathrm{U}_{24} \mathrm{Pp}_{12}$.

\section{S5. Density Measurements}

Density measurements were performed using an Anton Paar DMA 3400 M density meter.

Table S2. Results from density measurements of standards used in thermochemical calculations.

\begin{tabular}{|l|l|l|}
\hline Compound & $\mathrm{NaOH}_{(\mathrm{aq})}$ density $(\mathrm{g} / \mathrm{L})$ & $\mathrm{K}_{4} \mathrm{P}_{2} \mathrm{O}_{7(\mathrm{aq})}$ density $(\mathrm{g} / \mathrm{L})$ \\
\hline Solution \#1 & 1.04101 & 1.39016 \\
\hline Solution \#2 & 1.03296 & 1.20485 \\
\hline Solution \#3 & 1.02446 & 1.12504 \\
\hline Solution \#4 & 1.01597 & 1.08353 \\
\hline
\end{tabular}

Table S3. Results from density measurements of cluster solutions.

\begin{tabular}{ll}
\hline Compound & Density (g/L) \\
\hline LiNa- $U_{24} \mathrm{Pp}_{12}$ & 1.00910 \\
\hline
\end{tabular}




\section{S6. Thermographs}

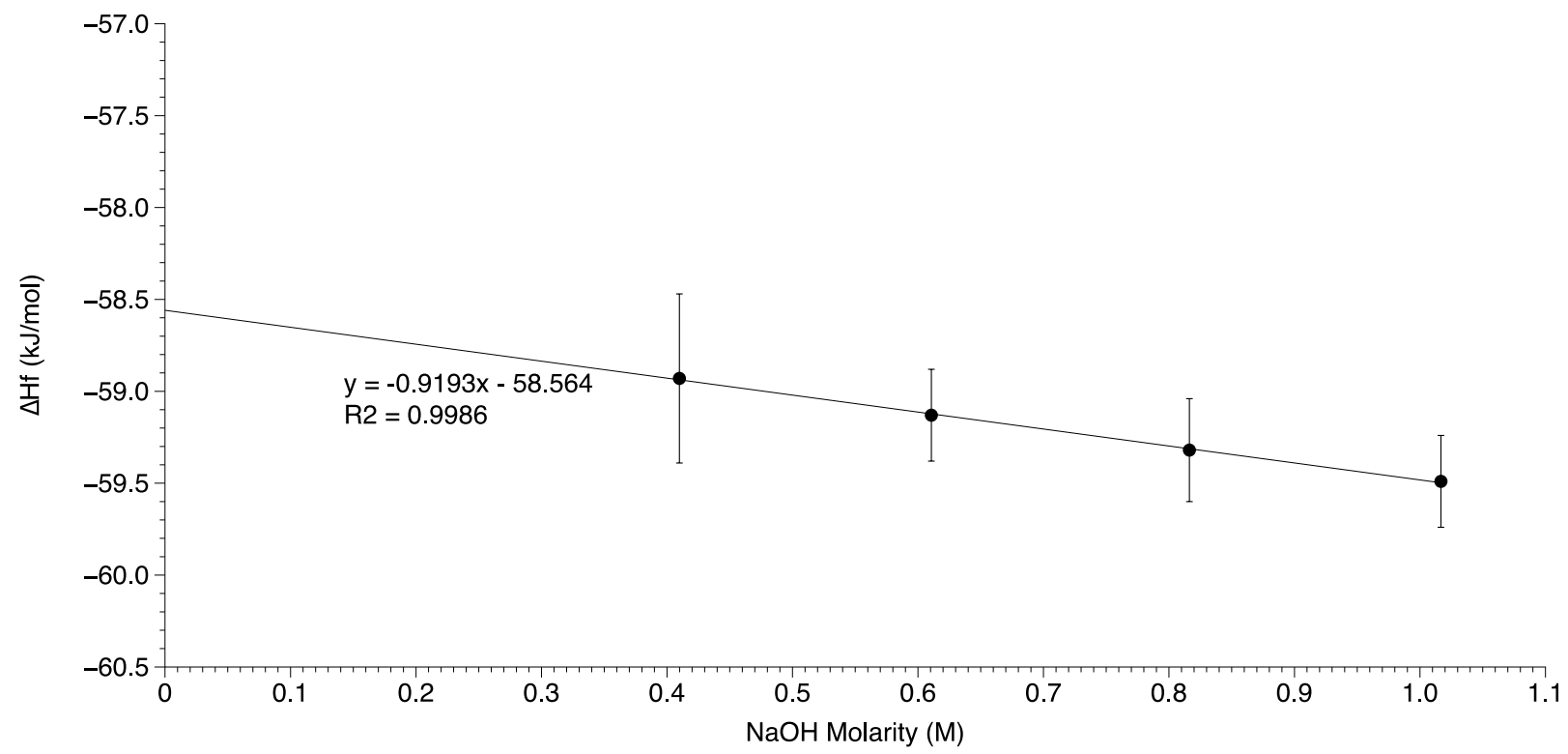

Figure S3. Thermograph for $\mathrm{NaOH}_{(\mathrm{aq})}$, used to extrapolate to the appropriate $\Delta H$ value for thermochemical calculations.

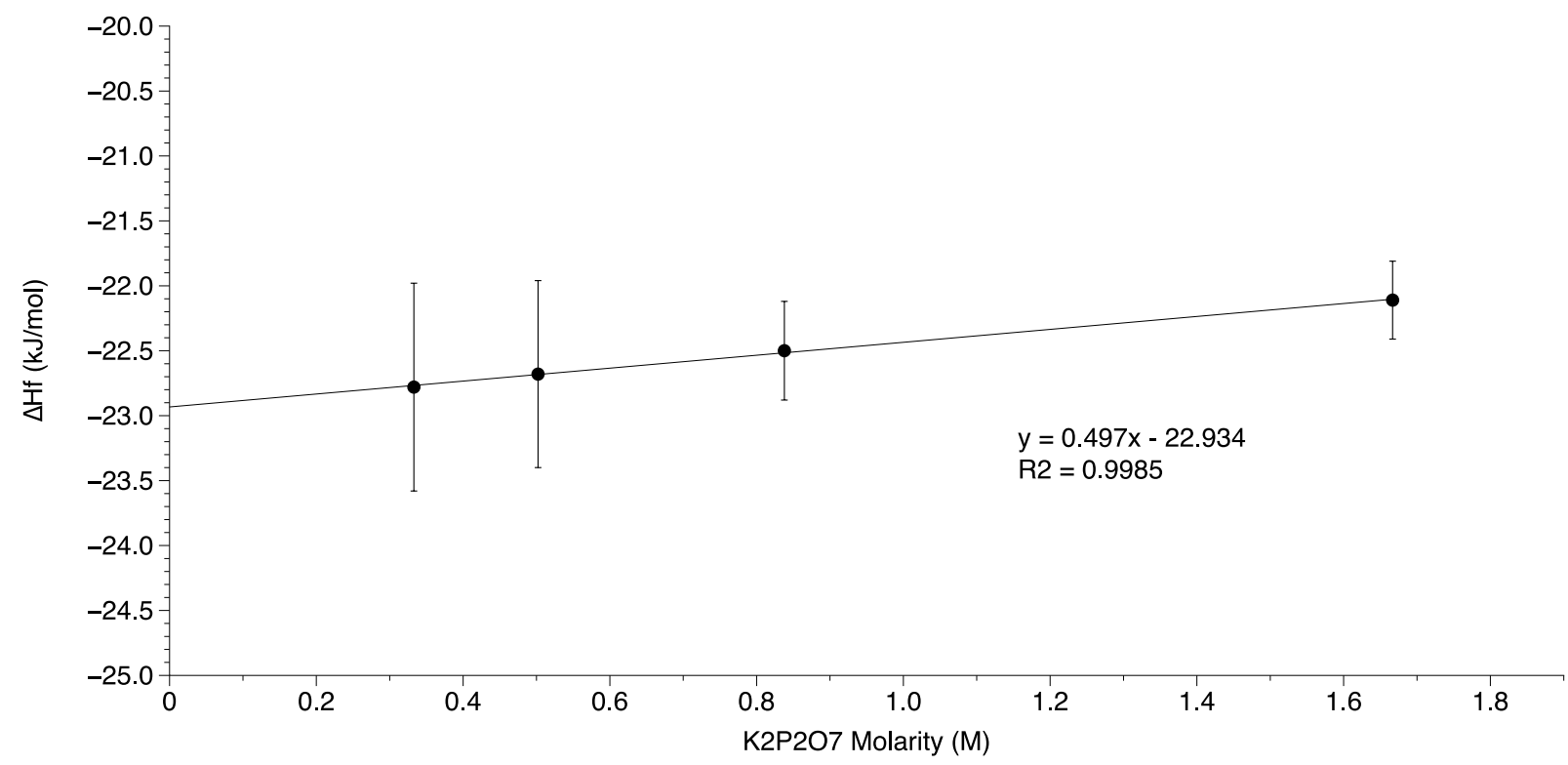

Figure S4. Thermograph for $\mathrm{K}_{4} \mathrm{P}_{2} \mathrm{O}_{7(\mathrm{aq})}$, used to extrapolate to the appropriate $\Delta H$ value for thermochemical calculations.

\section{S7. Thermochemical Cycles}

Table S4. Error is represented as two standard deviations from the mean. $\mathrm{n}=$ excess, $M=$ molarity. 


\begin{tabular}{|c|c|}
\hline Reaction & $\Delta H(\mathrm{~kJ} / \mathrm{mol})$ \\
\hline $\mathrm{Li}_{24} \mathrm{Na}_{24}\left[\left(\mathrm{UO}_{2} \mathrm{O}_{2}\right)_{24}\left(\mathrm{P}_{2} \mathrm{O}_{7}\right)_{12}\right]_{(\mathrm{aq})}+96 \mathrm{HCl}_{(\mathrm{aq})}=24 \mathrm{Li}^{+}{ }_{(\mathrm{aq}, \text { dil) }}$ & \\
\hline $\begin{array}{l}24 \mathrm{Na}^{+}{ }_{\text {aq, dil })}+24 \mathrm{UO}_{2}{ }^{2+}{ }_{(\mathrm{aq}, \text { dil })}+24 \mathrm{H}_{2} \mathrm{O}_{(\mathrm{l})}+12 \mathrm{O}_{2(\mathrm{~g})}+12 \\
\mathrm{H}_{4} \mathrm{P}_{2} \mathrm{O}_{7}+96 \mathrm{Cl}^{-}{ }_{(\mathrm{aq}, \text { dil })}\end{array}$ & $\Delta H_{1}=\Delta H_{\mathrm{ds}}=-555.4 \pm 32.0$ \\
\hline $\begin{array}{l}\mathrm{UO}_{2}\left(\mathrm{NO}_{3}\right)_{2(\mathrm{aq}, \text { dil) }}+2 \mathrm{HCl}_{(\mathrm{aq})}=\mathrm{UO}_{2}^{2+}{ }_{(\mathrm{aq}, \text { dil })}+\mathrm{Cl}_{(\mathrm{aq})}^{-}+\mathrm{NO}_{3}^{-} \\
\left(\text {aq, dil) }+\mathrm{HCl}_{(\mathrm{aq})}+\mathrm{HNO}_{3(\mathrm{aq}, \mathrm{dil})}\right.\end{array}$ & $\Delta H_{2}=15.3 \pm 1.3^{2}$ \\
\hline $\mathrm{LiOH}_{(\mathrm{aq}, \text { dil) }}+\mathrm{HCl}_{(\mathrm{aq})}=\mathrm{Li}_{(\mathrm{aq}, \text { dil) }}+\mathrm{Cl}_{(\mathrm{aq}, \text { dil) }}^{-}+\mathrm{H}_{2} \mathrm{O}_{(\mathrm{l})}$ & $\Delta H_{3}=-58.9 \pm 2.6^{2}$ \\
\hline $\mathrm{NaOH}_{(\text {aq, dil) }}+\mathrm{HCl}_{(\mathrm{aq})}=\mathrm{Na}_{(\text {(aq, dil) }}^{+}+\mathrm{Cl}^{-}{ }_{(\mathrm{aq}, \text { dil) }}+\mathrm{H}_{2} \mathrm{O}_{(\mathrm{l})}$ & $\Delta H_{4}=-58.7 \pm 0.5$ \\
\hline $\begin{array}{l}\mathrm{K}_{4} \mathrm{P}_{2} \mathrm{O}_{7(\text { aq, dil) }}+4 \mathrm{HCl}_{(\mathrm{aq})}=4 \mathrm{~K}_{(\mathrm{aq}, \text { dil) }}^{+}+\mathrm{H}_{4} \mathrm{P}_{2} \mathrm{O}_{7 \text { aq, dil) }}+4 \mathrm{Cl}^{-} \\
\text {(aq, dil) }\end{array}$ & $\Delta H_{5}=-22.9 \pm 0.5$ \\
\hline $\mathrm{UO}_{2}\left(\mathrm{NO}_{3}\right)_{2(\mathrm{aq}, 1 \mathrm{M})}=\mathrm{UO}_{2}\left(\mathrm{NO}_{3}\right)_{2(\mathrm{aq}, \mathrm{dil})}$ & $\begin{array}{l}\Delta H_{6}=-2.76 \times(1-M) \pm(-2.76 \times(1-M)) \times \\
((1 / 0.9901-1) / 4)^{-1 *}\end{array}$ \\
\hline $\mathrm{LiOH}_{(\mathrm{aq}, 1 \mathrm{M})}=\mathrm{LiOH}_{(\mathrm{aq}, \mathrm{dil})}$ & $\begin{array}{l}\Delta H_{7}=-1.30 \times(1-M) \pm(-1.30 \times(1-M)) \times \\
((1 / 0.9961-1) / 4)^{-1 *}\end{array}$ \\
\hline $\mathrm{NaOH}_{(\mathrm{aq}, 1 \mathrm{M})}=\mathrm{NaOH}_{(\mathrm{aq}, \mathrm{dil})}$ & $\begin{array}{l}\Delta H_{8}=-0.92 \times(1-M) \pm(-0.92 \times(1-M)) \times \\
((1 / 0.9986-1) / 3)^{-1 *}\end{array}$ \\
\hline $\mathrm{K}_{4} \mathrm{P}_{2} \mathrm{O}_{7(\mathrm{aq}, 1 \mathrm{M})}=\mathrm{K}_{4} \mathrm{P}_{2} \mathrm{O}_{7 \text { (aq, dil) }}$ & $\begin{array}{l}\Delta H_{9}=0.50 \times(1-M) \pm(0.50 \times(1-M)) \times \\
((1 / 0.9985-1) / 4)^{-1 *}\end{array}$ \\
\hline $\begin{array}{l}\mathrm{UO}_{2}\left(\mathrm{NO}_{3}\right)_{2} \cdot 6 \mathrm{H}_{2} \mathrm{O}_{(\mathrm{c})}+\mathrm{n} \mathrm{H} \mathrm{H}_{2} \mathrm{O}=\mathrm{UO}_{2}\left(\mathrm{NO}_{3}\right)_{2(\mathrm{aq})}+(\mathrm{n}+6) \\
\mathrm{H}_{2} \mathrm{O}_{(\mathrm{I})}\end{array}$ & $\Delta H_{10}=-7.39 \pm 0.50^{2}$ \\
\hline $\mathrm{Na}_{2} \mathrm{H}_{2} \mathrm{P}_{2} \mathrm{O}_{2(\mathrm{~s})}+\mathrm{n} \mathrm{H}_{2} \mathrm{O}_{(\mathrm{l})}=\mathrm{Na}_{2} \mathrm{H}_{2} \mathrm{P}_{2} \mathrm{O}_{2(\mathrm{aq})}+\mathrm{n} \mathrm{H}_{2} \mathrm{O}_{(\mathrm{l})}$ & $\Delta H_{11}=\Delta H_{\text {diss }}\left(\mathrm{Na}_{2} \mathrm{H}_{2} \mathrm{P}_{2} \mathrm{O}_{2(\mathrm{~s})}\right)=7.66 \pm 0.15$ \\
\hline
\end{tabular}




\begin{tabular}{|c|c|}
\hline $\mathrm{U}_{(\mathrm{s})}+\mathrm{N}_{2(\mathrm{~g})}+7 \mathrm{O}_{2(\mathrm{~g})}+6 \mathrm{H}_{2(\mathrm{~g})}=\mathrm{UO}_{2}\left(\mathrm{NO}_{3}\right)_{2} \cdot 6 \mathrm{H}_{2} \mathrm{O}_{(\mathrm{c})}$ & $\begin{array}{l}\Delta H_{12}=\Delta H_{\mathrm{f}}{ }^{\circ}\left(\mathrm{UO}_{2}\left(\mathrm{NO}_{3}\right)_{2} \cdot 6 \mathrm{H}_{2} \mathrm{O}_{(\mathrm{c})}\right)=- \\
3,167.5 \pm 1.5^{3}\end{array}$ \\
\hline $\mathrm{Li}_{(\mathrm{s})}+1 / 2 \mathrm{O}_{2(\mathrm{~g})}+1 / 2 \mathrm{H}_{2(\mathrm{~g})}=\mathrm{LiOH}_{(\mathrm{aq})}$ & $\Delta H_{13}=\Delta H_{f}^{\circ}\left(\operatorname{LiOH}_{(\mathrm{aq})}\right)=-508.4 \pm 0.1^{4}$ \\
\hline $\mathrm{Na}_{(\mathrm{s})}+1 / 2 \mathrm{O}_{2(\mathrm{~g})}+1 / 2 \mathrm{H}_{2(\mathrm{~g})}=\mathrm{NaOH}_{(\mathrm{aq})}$ & $\Delta H_{14}=\Delta H_{\mathrm{f}}^{\circ}\left(\mathrm{NaOH}_{(\mathrm{aq})}\right)=-470.4 \pm 0.1^{5}$ \\
\hline $4 \mathrm{~K}_{(\mathrm{s})}+2 \mathrm{P}_{(\mathrm{s})}+31 / 2 \mathrm{O}_{2(\mathrm{~g})}=\mathrm{K}_{4} \mathrm{P}_{2} \mathrm{O}_{7(\mathrm{aq})}$ & $\begin{array}{l}\Delta H_{15}=\Delta H_{f}^{\circ}\left(\mathrm{K}_{4} \mathrm{P}_{2} \mathrm{O}_{7 \text { (aq) }}\right)=-3235.5 \pm \\
0.16^{6}\end{array}$ \\
\hline $1 / 2 \mathrm{H}_{2(\mathrm{~g})}+1 / 2 \mathrm{Cl}_{2(\mathrm{~g})}=\mathrm{HCl}_{(\mathrm{aq})}$ & $\Delta H_{16}=\Delta H_{\mathrm{f}}^{\circ}\left(\mathrm{HCl}_{(\mathrm{aq})}\right)=-166.266 \pm 0.1^{7}$ \\
\hline $\mathrm{H}_{2(\mathrm{~g})}+1 / 2 \mathrm{O}_{2(\mathrm{~g})}=\mathrm{H}_{2} \mathrm{O}_{(\mathrm{l})}$ & $\Delta H_{17}=\Delta H_{f}^{0}\left(H_{2} \mathrm{O}_{(I)}\right)=-285.8 \pm 0.1^{8}$ \\
\hline $\mathrm{K}_{(\mathrm{s})}=\mathrm{K}^{+}$(aq) & $\Delta H_{18}=\Delta H_{f}^{\circ}\left(\mathrm{K}^{+}{ }_{(\mathrm{aq})}\right)=-252.14 \pm 0.08^{9}$ \\
\hline $1 / 2 \mathrm{~N}_{2(\mathrm{~g})}+11 / 2 \mathrm{O}_{2(\mathrm{~g})}=\mathrm{NO}_{3(\mathrm{aq})}^{-}$ & $\Delta H_{19}=\Delta H_{\mathrm{f}}^{\circ}\left(\mathrm{NO}_{3_{(\mathrm{aq})}^{-}}\right)=-206.85 \pm 0.4^{5}$ \\
\hline $1 / 2 \mathrm{H}_{2(\mathrm{~g})}+1 / 2 \mathrm{~N}_{2(\mathrm{~g})}+11 / 2 \mathrm{O}_{2(\mathrm{~g})}=\mathrm{HNO}_{3(\mathrm{aq})}$ & $\Delta H_{20}=\Delta H_{f}^{\circ}\left(\mathrm{HNO}_{3 \text { (aq) }}\right)=-206.85 \pm 0.4^{5}$ \\
\hline $1 / 2 \mathrm{Cl}_{2(\mathrm{~g})}=\mathrm{Cl}_{(\mathrm{aq})}^{-}$ & $\Delta H_{21}=\Delta H_{f}^{\circ}\left(\mathrm{Cl}_{(\mathrm{aq})}^{-}\right)=-166.266 \pm 0.1^{7}$ \\
\hline $2 \mathrm{Na}_{(\mathrm{s})}+2 \mathrm{H}_{2(\mathrm{~g})}+2 \mathrm{P}_{(2)}+3 \frac{1}{2} \mathrm{O}_{2(\mathrm{~g})}=\mathrm{Na}_{2} \mathrm{H}_{2} \mathrm{P}_{2} \mathrm{O}_{7(\mathrm{~s})}$ & $\begin{array}{l}\Delta H_{22}=\Delta H_{f}^{\circ}\left(\mathrm{Na}_{2} \mathrm{H}_{2} \mathrm{P}_{2} \mathrm{O}_{2(\mathrm{~s})}\right)=-2,781.1 \pm \\
4.2^{10}\end{array}$ \\
\hline $\begin{array}{l}24 \mathrm{Li}_{(\mathrm{s})}+24 \mathrm{U}_{(\mathrm{s})}+60 \mathrm{O}_{2(\mathrm{~g})}+12 \mathrm{H}_{2(\mathrm{~g})}= \\
\mathrm{Li}_{24}\left[\mathrm{UO}_{2} \mathrm{O}_{2} \mathrm{OH}\right]_{24(\text { (aq) }}\end{array}$ & $\Delta H_{23}=\Delta H_{\mathrm{f}}{ }^{\circ}\left(\mathrm{Li}-\mathrm{U}_{24(\mathrm{aq})}\right)=-44,377.9 \pm 24.1$ \\
\hline
\end{tabular}




\begin{tabular}{|c|c|}
\hline $\begin{array}{l}24 \mathrm{Li}_{(\mathrm{s})}+24 \mathrm{Na}_{(\mathrm{s})}+24 \mathrm{U}_{(\mathrm{s})} 24 \mathrm{P}_{(\mathrm{s})}+90 \mathrm{O}_{2(\mathrm{~g})}= \\
\mathrm{Li}_{24} \mathrm{Na}_{24}\left[\left(\mathrm{UO}_{2} \mathrm{O}_{2}\right)_{24}\left(\mathrm{P}_{2} \mathrm{O}_{7}\right)_{12}\right]_{(\mathrm{aq})}\end{array}$ & $\begin{array}{l}\Delta H_{24}=\Delta H_{\mathrm{f}}^{\circ}\left(\text { LiNa- } \mathrm{U}_{24} \mathrm{Pp}_{12(\mathrm{aq})}\right)=-\Delta H_{1}+ \\
24 \Delta H_{2}+24 \Delta H_{3}+24 \Delta H_{4}+12 \Delta H_{5}+24 \\
\Delta H_{6}+24 \Delta H_{7}+24 \Delta H_{8}+12 \Delta H_{9}+24 \\
\Delta H_{10}++24 \Delta H_{12}+24 \Delta H_{13}+24 \Delta H_{14}+ \\
12 \Delta H_{15}+24 \Delta H_{16}-168 \Delta H_{17}-48 \Delta H_{18} \\
-24 \Delta H_{19}-24 \Delta H_{20}-24 \Delta H_{21}=-70.321 .7 \\
\pm 35.8\end{array}$ \\
\hline $\begin{array}{l}\mathrm{Li}_{24} \mathrm{Na}_{24}\left[\left(\mathrm{UO}_{2} \mathrm{O}_{2}\right)_{24}\left(\mathrm{P}_{2} \mathrm{O}_{7}\right)_{12}\right]_{(\mathrm{aq})}+24+\mathrm{H}_{2} \mathrm{O}_{(\mathrm{l})}= \\
\mathrm{Li}_{24}\left[\mathrm{UO}_{2} \mathrm{O}_{2} \mathrm{OH}\right]_{24(\mathrm{aq})}+12 \mathrm{Na}_{2} \mathrm{H}_{2} \mathrm{P}_{2} \mathrm{O}_{7(\mathrm{aq})}\end{array}$ & $\begin{array}{l}\Delta H_{25}=\Delta H_{\text {con }}^{\circ}=\Delta H_{23}+12 \Delta H_{11}+\Delta H_{22}- \\
\Delta H_{24}-24 \Delta H_{17}=-637.7 \pm 14.5\end{array}$ \\
\hline
\end{tabular}

*The enthalpy value and the error is derived from the thermographs (Figure S3-S4) where $M$ is molarity and error is computed using Equation S1. Error $=\Delta H x \sqrt{\frac{\frac{1}{\left(R^{2}\right)^{2}}-1}{4}}$

Equation S1

\section{S8. In situ Raman Spectroscopy}

Raman data from heat ramping experiments were collected using a Bruker Sentinel system equipped with a fibre optic probe, thermoelectric cooled CCD detector, and a $785 \mathrm{~nm}$ excitation source. Spectra were collected over the range of $80-3200 \mathrm{~cm}^{-1}$ using ten 60 -second exposures at $400 \mathrm{~mW}$ laser power. Baseline corrections were performed on each data set using the Sonneveld and Visser algorithm implemented in MATLAB. ${ }^{11}$

\section{S9. Small Angle X-ray Scattering}

A Bruker Nanostar equipped with a $\mathrm{Cu}$ microfocus sources, Montel multilayer optics, and a HiSTAR multiwire detector was used for SAXS measurements. The samples were loaded into Kapton capillaries and the ends of the capillaries were sealed with epoxy. Water loaded into identical capillaries was used for background measurements and scattering data were collected for water with and without a glassy carbon standard for the correction to absolute intensity and background subtraction. Each sample was collected for 2 hours. 


\section{S10. Pictures of Crystals}

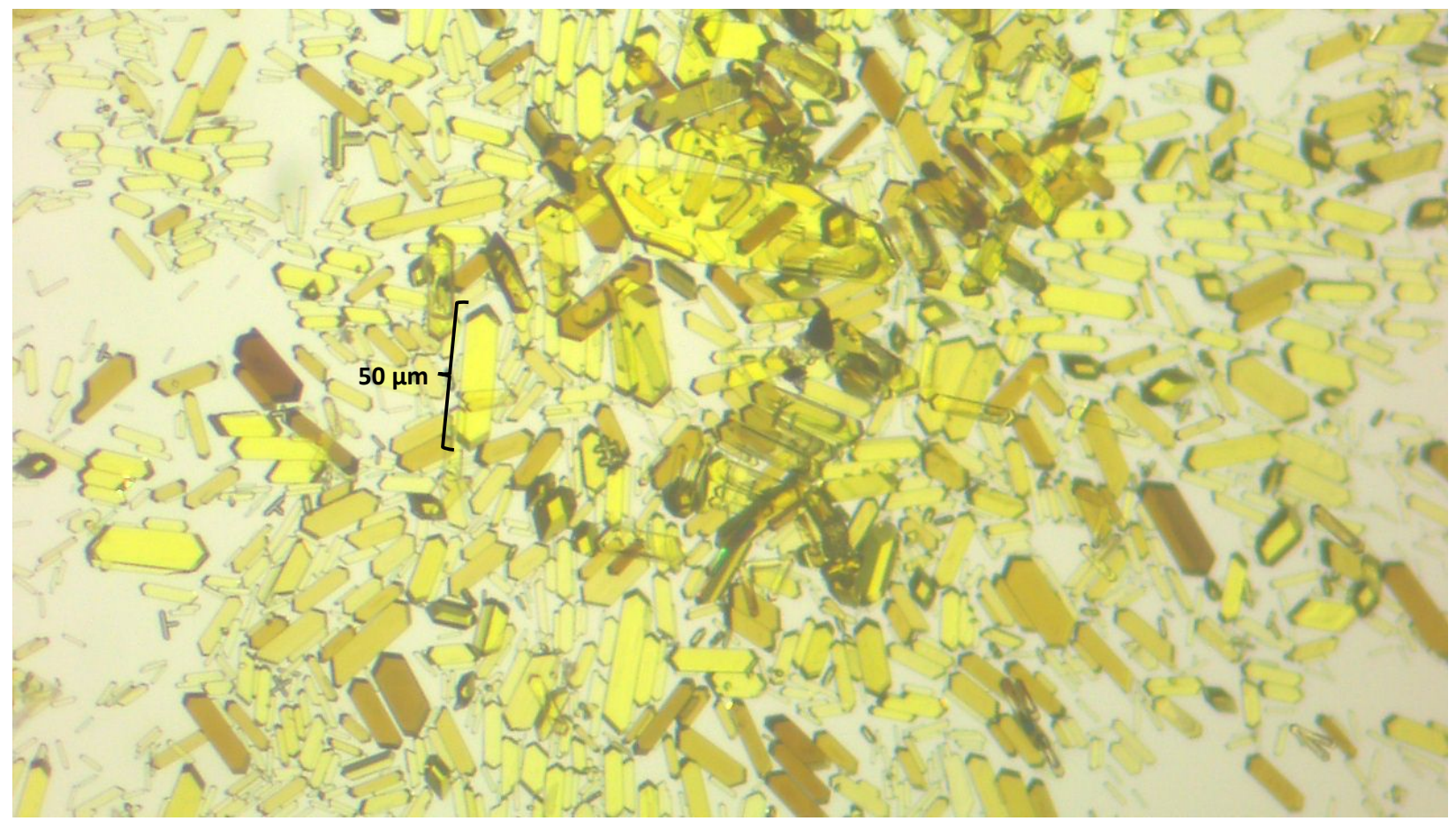

Figure S5. Co-crystallized $\mathrm{U}_{24} \cdot \mathrm{U}_{24} \mathrm{Pp}_{12}$ as thin double-edge sword shaped crystals. Picture taken from solution heated to $110^{\circ} \mathrm{C}$. Crystals ranged from $20 \mu \mathrm{m}$ to about $100 \mu \mathrm{m}$ in length at the longest dimension 


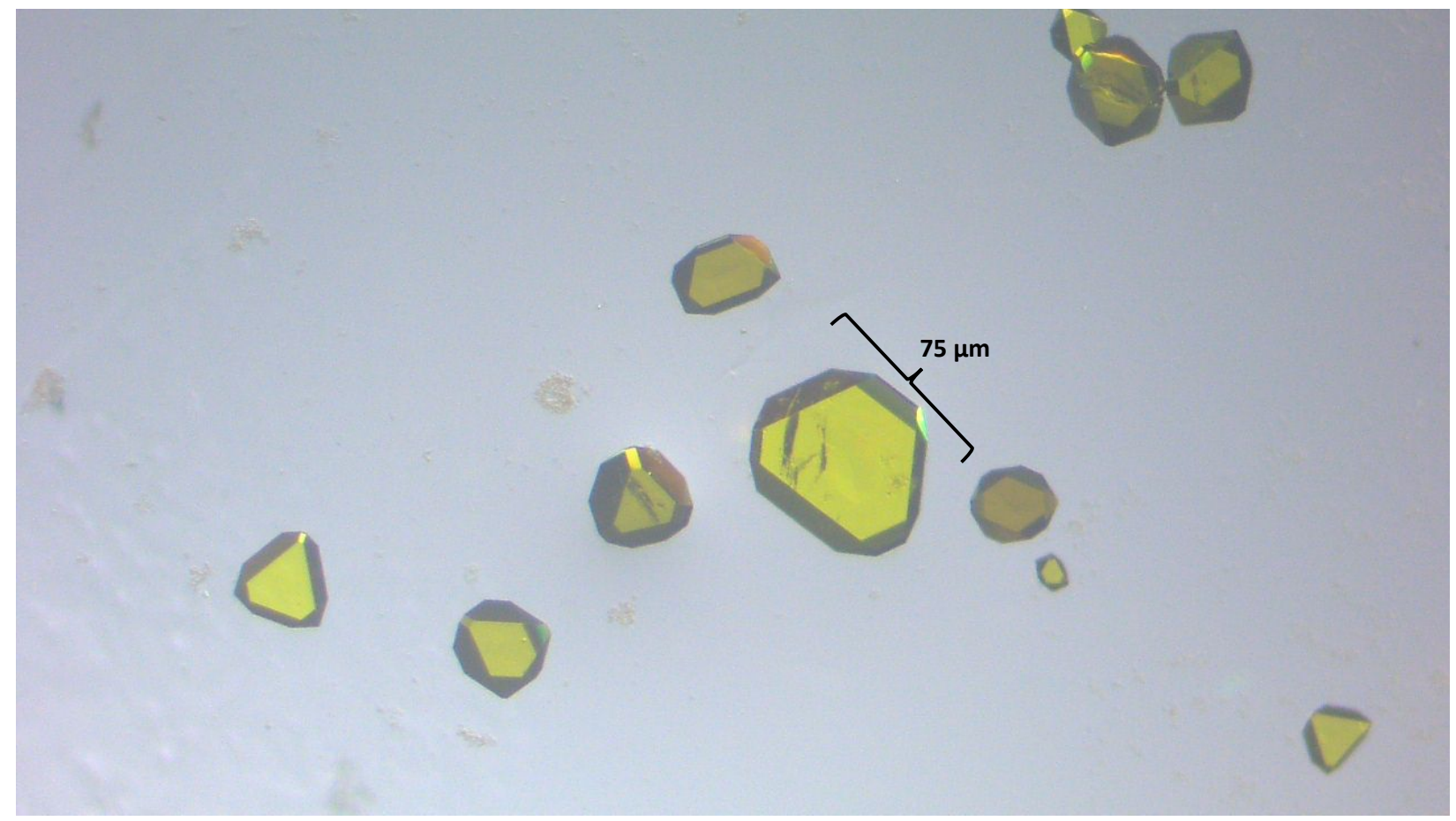

Figure S6. LiNa- $U_{24}$ crystallized as octahedral shaped crystals that ranged from $15 \mu \mathrm{m}$ to $75 \mu \mathrm{m}$ in length. Picture taken from solution that was headed to $135^{\circ} \mathrm{C}$. 
S11. Nuclear Magnetic Resonance

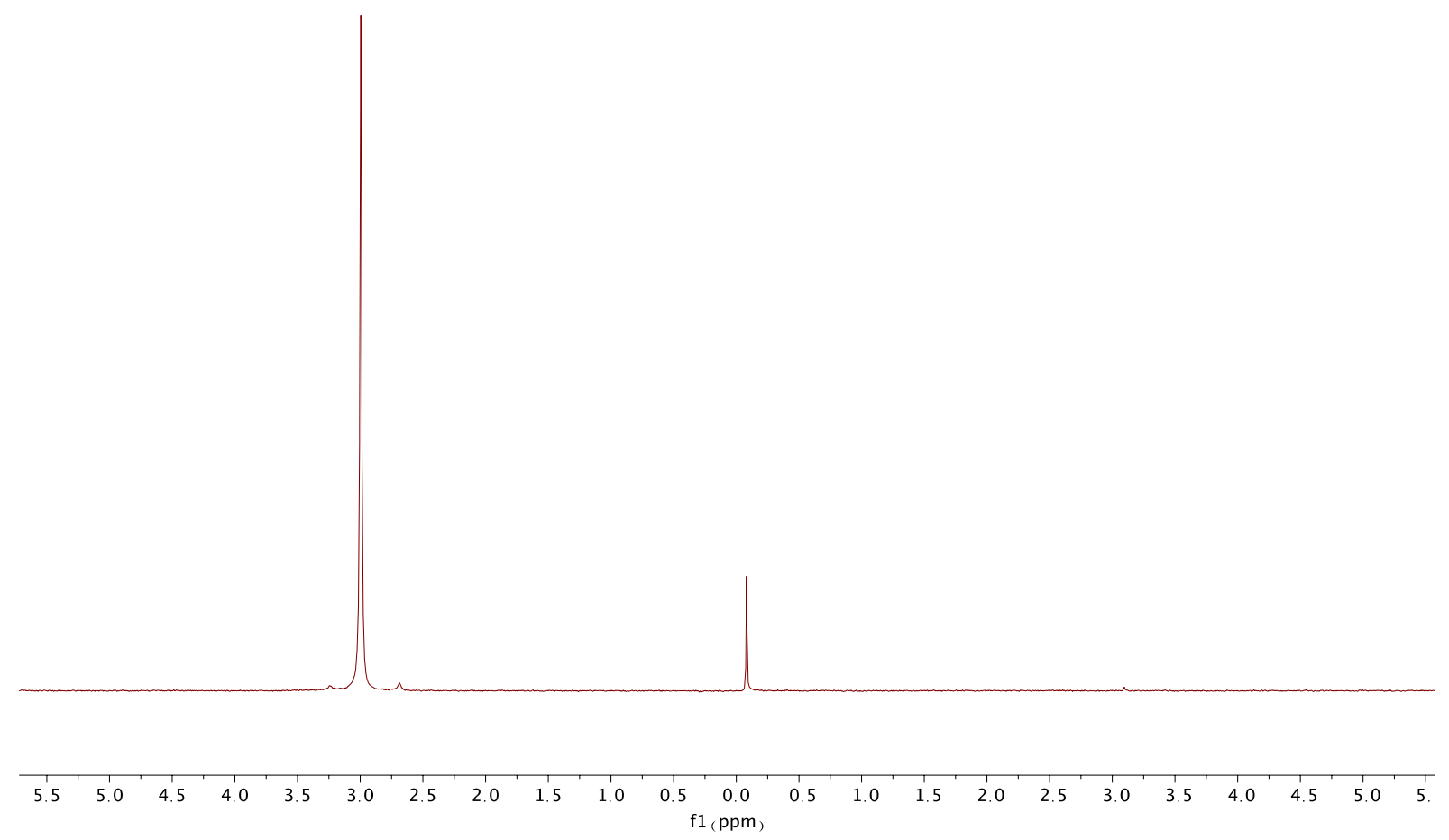

Figure S7. ${ }^{31} \mathrm{P} N M R$ of $\mathrm{LiNa}-\mathrm{U}_{24} \mathrm{Pp}_{12}$ solution used for calorimetry studies. 


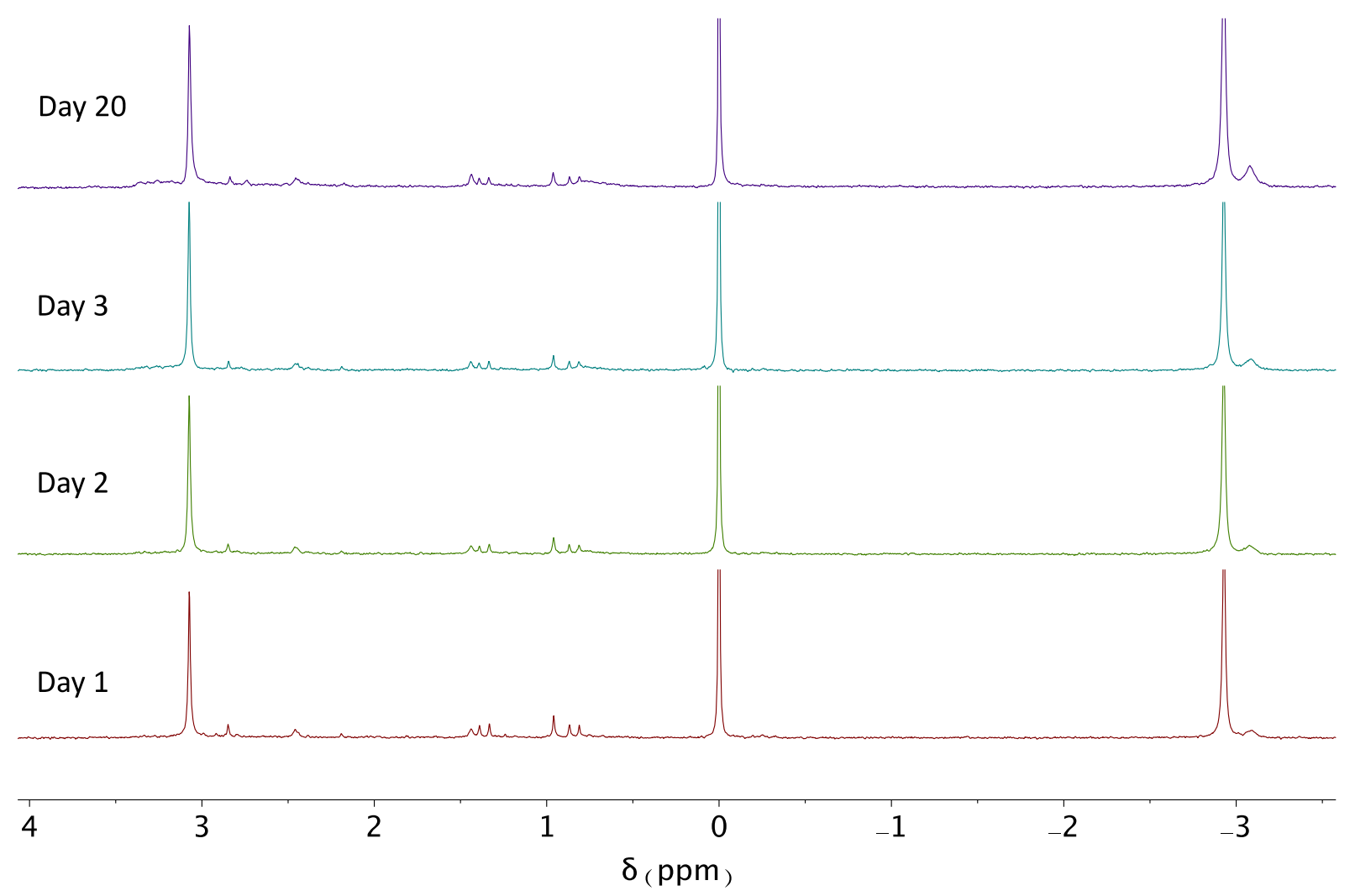

Figure S8. A heated solution of $\mathrm{U}_{24} \mathrm{Pp}_{12}$ was monitored periodically over a span of 20 days with no signs of change to the ${ }^{31} \mathrm{P}$ NMR spectra.

\section{S12. Separations}

About $0.5 \mathrm{~mL}$ of solution were placed into a Amicon Ultra- $0.53 \mathrm{~K}$ centrifugal filter device, and the filter was subsequently placed into a filtrate collection tube before centrifuging the tube for 20 minutes at 7,000 RPM. The resulting filtrate was collected and placed into a new filter and the centrifugation was repeated. The process was repeated again, so that the solution had been filtrated three times in total. 


\section{S13. Crystallographic Data}

Table S5. Crystal data and details of the structure determination for LiNa- $\mathrm{U}_{24}$.

\begin{tabular}{|c|c|}
\hline \multicolumn{2}{|c|}{ Crystal Data } \\
\hline Formula Weight & 8583.71 \\
\hline Crystal System & Tetragonal \\
\hline Space group & $14 / \mathrm{m}$ \\
\hline$a(\AA)$ & $19.434(3)$ \\
\hline$c(\AA)$ & $21.060(4)$ \\
\hline$V\left(\AA^{3}\right)$ & $7954(3)$ \\
\hline$Z$ & 2 \\
\hline Absorption coefficient $\left(\mathrm{mm}^{-1}\right)$ & 24.468 \\
\hline$F(000)$ & 7286 \\
\hline Crystal Size [mm] & $0.05 \times 0.05 \times 0.05$ \\
\hline \multicolumn{2}{|c|}{ Data Collection } \\
\hline Temperature (K) & 100 \\
\hline Radiation $(\AA)$ & $\mathrm{MoK}_{a} ; 0.71073$ \\
\hline$\theta_{\min } \rightarrow \theta_{\max }\left({ }^{\circ}\right)$ & $1.4,27.5$ \\
\hline$H, k, I$ & $-25 \rightarrow 25 ;-25 \rightarrow 25 ;-27 \rightarrow 27$ \\
\hline Total Reflections & 47736 \\
\hline Unique Reflections & 4731 \\
\hline$R_{\text {int }}$ & 0.056 \\
\hline Observed Reflections $[I>2 \sigma(I)]$ & 4018 \\
\hline \multicolumn{2}{|c|}{ Refinement } \\
\hline Number of Parameters & 223 \\
\hline$R_{1}$ & 0.0364 \\
\hline$w R_{2}$ & 0.1110 \\
\hline$S$ & 1.12 \\
\hline Weight scheme & $w=1 /\left[\sigma^{2} F_{o}^{2}+(0.1 P)^{2}\right], P=\left(F_{o}^{2}+2 F_{c}^{2}\right) / 3$ \\
\hline
\end{tabular}




\begin{tabular}{|c|c|}
\hline Max. and Av. Shift/Err & $0.00,0.00$ \\
\hline$\Delta \rho_{\min } / \Delta \rho_{\max }\left(\AA^{3}\right)$ & $-2.01,3.88$ \\
\hline
\end{tabular}

\section{Reference}

1. Dembowski, M.; Colla, C. A.; Hickam, S.; Oliveri, A. F.; Szymanowski, J. E. S.; Oliver, A. G.; Casey, W. H.; Burns, P. C., Hierarchy of Pyrophosphate-Functionalized Uranyl Peroxide Nanocluster Synthesis. Inorganic Chemistry 2017, 56 (9), 5478 5487.

2. Traustason, H.; Bell, N. L.; Caranto, K.; Auld, D. C.; Lockey, D. T.; Kokot, A.; Szymanowski, J. E. S.; Cronin, L.; Burns, P. C., Reactivity, Formation, and Solubility of Polyoxometalates Probed by Calorimetry. Journal of the American Chemical Society 2020, 142 (48), 20463-20469.

3. Grenthe, I.; Fuger, J.; Konings, R. J. M.; Lemire, R. J.; Muller, A. B.; Nguyen-Trung Cregu, C.; Wanner, H., Chemical Thermodynamics of Uranium, 1. Nuclear Energy Agency: North-Holland Amsterdam, 1992.

4. Monnin, C.; Dubois, M., Thermodynamics of the $\mathrm{LiOH}+\mathrm{H} 2 \mathrm{O}$ system. Journal of Chemical and Engineering Data 2005, $50(4), 1109-1113$.

5. $\quad$ Cox, J. D.; Wagman, D. D.; Medvedev, V. A. In CODATA key values for thermodynamics 1989.

6. Luff, B. B.; Reed, R. B., Standard enthalpy of formation of tetrapotassium pyrophosphate. Journal of Chemical \& Engineering Data 1979, 24 (3), 227-228.

7. Konings, R. J. M.; Cordfunke, E. H. P.; Ouweltjes, W., THE STANDARD ENTHALPIES OF FORMATION OF HYDROXIDES .2. THE ALKALI HYDROXIDES CSOH AND KOH. Journal of Chemical Thermodynamics 1988, 20 (7), 777-780.

8. Robie, R. A.; Hemingway, B. S., Thermodynamic properties of minerals and related substances at $298.15 \mathrm{~K}$ and $1 \mathrm{bar}$ (10^5 pascals) pressure and at higher temperatures. U.S. Geological Survey 1995, 461.

9. Rumble, J. R., CRC Handbook of Chemistry and Physics. 101st Edition ed.; CRC Press/Taylor \& Francis: Boca Raton, FL, 2020.

10. Irving, R. J.; McKerrell, H., Standard heats of formation of two sodium pyrophosphates, sodium trimetaphosphate, and sodium tetrametaphosphate. Transactions of the Faraday Society 1968, 64 (0), 879-882.

11. Sonneveld, E. J.; Visser, J. W., AUTOMATIC COLLECTION OF POWDER DATA FROM PHOTOGRAPHS. Journal of Applied Crystallography 1975, 8 (FEB1), 1-7. 